\title{
Development of polarized HD target for SPring-8/LEPS experiment
}

Yuka Yanai*

Research Center for Nuclear Physics, Osaka university, Ibaraki, Osaka, 567-0047, Japan

E-mail: vanaiercnp.osaka-u.ac.ip

Hideki Kohri

Research Center for Nuclear Physics, Osaka university, Ibaraki, Osaka, 567-0047, Japan

E-mail: kohriercnp.osaka-u.ac.ip

\section{Masaru Yosoi}

Research Center for Nuclear Physics, Osaka university, Ibaraki, Osaka, 567-0047, Japan

E-mail: yosoidercnp.osaka-u.ac.7p

\section{Tomoaki Hotta}

Research Center for Nuclear Physics, Osaka university, Ibaraki, Osaka, 567-0047, Japan

E-mail: hottadrenp.osaka-u.ac.ip

\section{Hitoshi Katsuragawa}

Research Center for Nuclear Physics, Osaka university, Ibaraki, Osaka, 567-0047, Japan E-mail: katsuragdrcnp.osaka-u.ac.jp

\section{Takeshi Ohta}

Depertment of Radiology, The University of Tokyo Hospital, 7-3-1 Hongo Bunkyo-ku Tokyo 113-8655 Japan

E-mail: takeshidrcnp.osaka-u.ac.jp

\section{Shintaro Tanaka}

Research Center for Nuclear Physics, Osaka university, Ibaraki, Osaka, 567-0047, Japan E-mail: stanakadrcnp.osaka-u.ac.jp

A polarized solid HD (Hydrogen - Deuteride) has been developed for near future photoproduction experiment at SPring-8/LEPS. The solid HD target is polarized by the static method in a strong magnetic field of 17 Tesla and low temperature of about $15 \mathrm{mK}$ at RCNP Osaka university. The solid HD target is transported from RCNP to SPring-8, under the condition of a magnetic field larger than $0.1 \mathrm{~T}$ for holding the polarization and low temperature lower than $4 \mathrm{~K}$, in which we use five cryostats. We have achieved a polarization degree of $44 \%$ and a relaxation time of 8 months for $\mathrm{H}$. We have recently succeeded in the connection among three cryostats used at SPring- 8 . After succeeding all the target transportations, we will start the physics experiment at SPring- 8 .

XVII International Workshop on Polarized Sources, Targets \& Polarimetry

16-20 October 2017

Kaist, South Korea

\footnotetext{
* Speaker.
} 


\section{Introduction}

We have studied hadron photoproduction at SPring-8. The unpolarized $\mathrm{LH}_{2}$ and $\mathrm{LD}_{2}$ targets have been used in the experiments so far. We have developed a new target, i.e., a polarized solid HD target for a new photoproduction experiment. We deal with five cryostats to make and transport the HD target and to carry out the experiments. This paper describes recent status of the project.

\subsection{Laser electron photon beam line at SPring-8 (LEPS)}

The LEPS collaboration has parformed the experiments of photo-nucleon and photo-nuclear reactions at SPring- 8 by using linearly polarized photons from Laser backward compton scattering[W, []]. Figure 1 shows the overall view of LEPS beam line at SPring-8. Various mesons and baryons are produced at LEPS. The photon beam with energies from 1.5 to $3.0 \mathrm{GeV}$ can be used. We have used the LEPS spectrometer to measure hadron. The LEPS forward spectrometer consists of a dipole magnet and a start counter, a vertex counter, three drift chambers, an aerogel cherenkov counter, and a time of flight hodoscope (TOF).

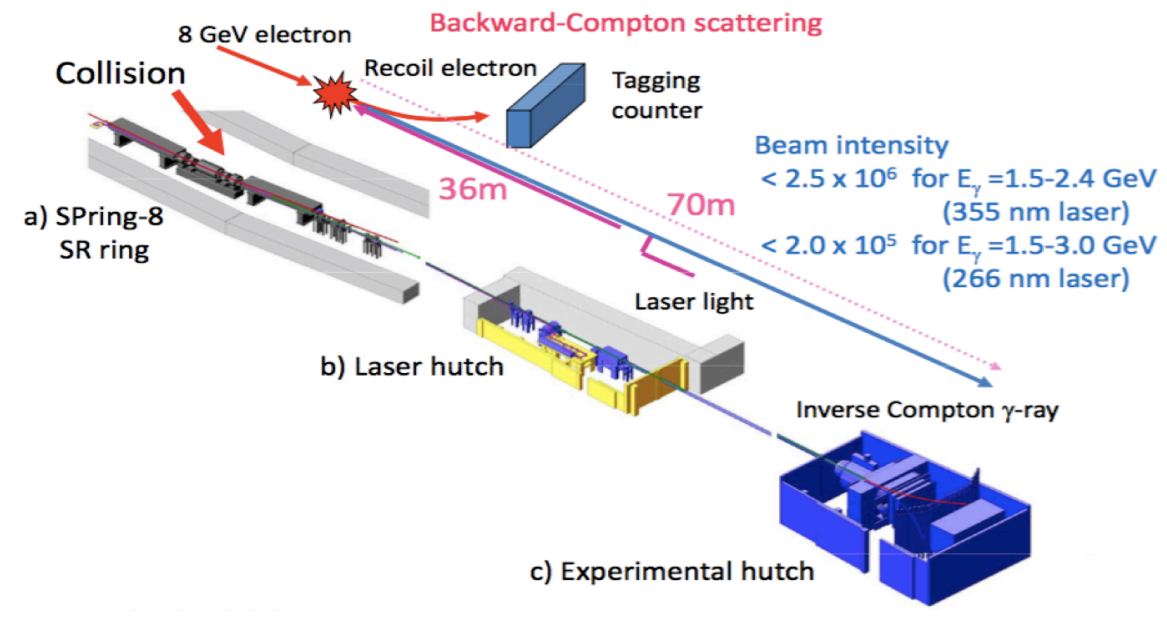

Figure 1: LEPS beam line at SPring-8. We use photon beams from Laser backward-Compton scattering. The $8 \mathrm{GeV}$ electrons and the UV laser beams collide in the SR ring of SPring-8. The produced high energy photon beams come to experimental hutch.

\subsection{Physics motivation of the experiment at LEPS with polarized target}

We plan to carry out experiments of hadron photoproduction by using both polarized photon beam and the polarized solid HD target in the near future. When longitudinally and transversely polarized targets are used in addition to linearly and circularly polarized photon beams, we can measure rich physics observables in hadron photoproduction [3], 团]. One of our main objectives is to measure double polarization observables in $\phi$ meson production to investigate the strangeness contents of the proton through the $s \bar{s}$ knock out process[[5]. 


\section{Polarized solid HD target}

The HD is a heteronuclear diatomic molecule consisting of hydrogen and deuterium. We use static polarization method using Brute-force[可] with strong magnetic field of 17 Tesla and low temperature of $\sim 15 \mathrm{mK}$. The polarized solid HD target was firstly proposed by Honig in 1967 [四]. The polarized solid HD target system was used for the actual experiments at LEGS [Q, Q $Q$ ] and GRAAL [ए0]. Figure 2 shows the target cell and the NMR coil. The Al wires are inserted to the target cell for cooling the solid HD ( $\mathrm{Al}$ wire is $\phi=50 \mu \mathrm{m}$ ). The capsule is made of Kel-F which does not include hydrogen materials. The target base is made of $\mathrm{Cu}$. The diameter of the target is $30 \mathrm{~mm}$ and the thickness is $50 \mathrm{~mm}$.

\subsection{The process from polarized HD production to experiment}

Since purity of commercial HD gas is about $96 \%\left(\mathrm{H}_{2} 2 \%, \mathrm{D}_{2} 2 \%\right)$, we purified the HD gas to $99.99 \%$ by using the RCNP distillation system [ए]]. The pure HD cannot be polarized easily. We add a small amount of ortho- $\mathrm{H}_{2}$ to shorten the relaxation time [12]. The orth- $\mathrm{H}_{2}$ converts to the para- $\mathrm{H}_{2}$ with lifetime of 6.3 days. Since the para- $\mathrm{H}_{2}$ does not interact with HD, the HD becomes spin-frozen. The polarization is kept even at B $\sim 1$ Tesla, $\mathrm{T} \sim 300 \mathrm{mK}[\mathrm{Q}]$. We operate five cryostats for making and moving the polarized solid HD target. We produce the target at RCNP and carry out experiment at SPring-8. Firstly the Dilution Refrigerator (DR) is used to polarize and freeze the solid HD target at B $=17 \mathrm{~T}, \mathrm{~T}=\sim 15 \mathrm{mK}$ at RCNP. Secondly the Transfer Cryostat 1 (TC1) is used to transport the HD target from the DR to the Storage Cryostat (SC) at $\mathrm{B}=0.1 \mathrm{~T}, \mathrm{~T}=4 \mathrm{~K}$. The SC is moved from RCNP to SPring- 8 at $\mathrm{B}=1 \mathrm{~T}, \mathrm{~T}=\sim 2 \mathrm{~K}$. In that process we use an iris for thermal shield(see figure 3). Figure 4 shows the connection of the Transfer Cryostat 2 (TC2) and the SC. The shield layer is cooled to $50 \mathrm{~K}$ by the first stage of a $4 \mathrm{~K}-\mathrm{GM}$ refrigerator. The TC2 has a $\mathrm{MgB}_{2}$ superconductive magnet. It induces a magnetic field of $0.15 \mathrm{~T}$ with a current of $40 \mathrm{~A}$. The critical temperature is $39 \mathrm{~K}$. Finally the HD target is taken out by the TC2 from the SC to the In

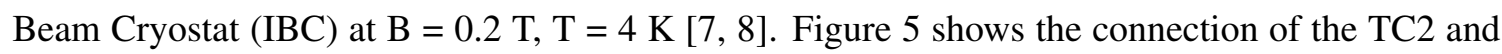
the IBC. The IBC is used in the experiment at $\mathrm{B}=1 \mathrm{~T}, \mathrm{~T}=300 \mathrm{mK}$.

(a) Target cell

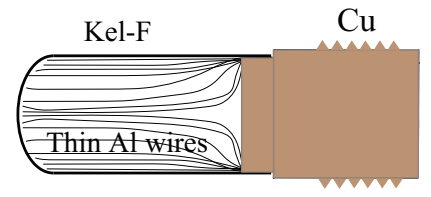

(b) Iris shutter and opener

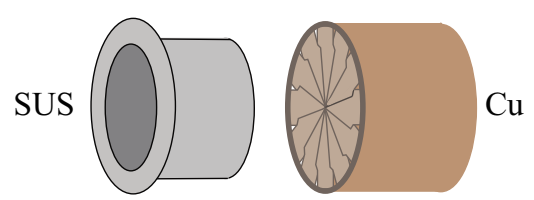

Figure 2: (a)The target cell and (b)Iris shutter and opener. The iris is attached to the tip of the TC2 shield layer to prevent radiation heats from entering to the target.

\subsection{NMR}

We use the portable NMR system in the measurement of the polarization. We have developed a portable NMR system [[13]]. The portable NMR system enables us to measure the polarization of the solid HD target during the transportation. It consists of a laptop PC, a PXI system, an attenuator, a 


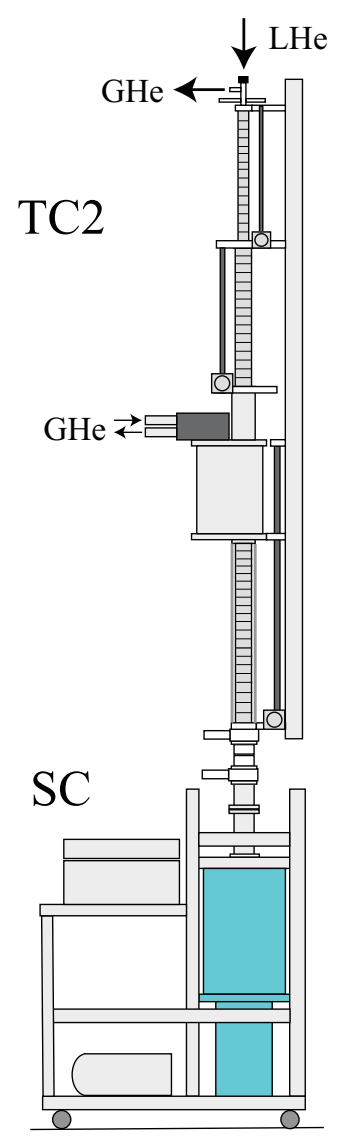

Figure 3: The connection of the TC2 and the SC. A bellow is used in the connecting part between the TC2 and the SC. The LHe is filled from the top of the TC2 and the He gas escapes from the side pipe. The TC2 moves vertically at this condition. The TC2 length is $4 \mathrm{~m}$, and the temperature of the target layer is 4.2 $\mathrm{K}$ cooled by LHe and the temperature of magnet layer is $\leq 30 \mathrm{~K}$ cooled by the second stage of a $4 \mathrm{~K}-\mathrm{GM}$ refrigerator.

IBC

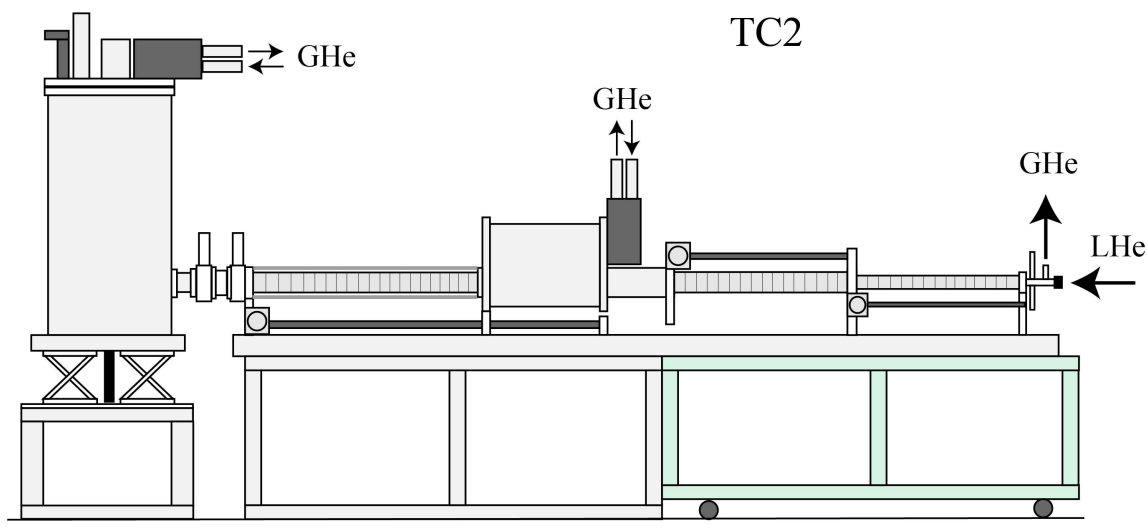

Figure 4: The connection of the IBC and the TC2. A bellow is used in the connecting part between the TC2 and the SC. The TC2 moves horizontally at this condition. 


\section{IBC TC2}

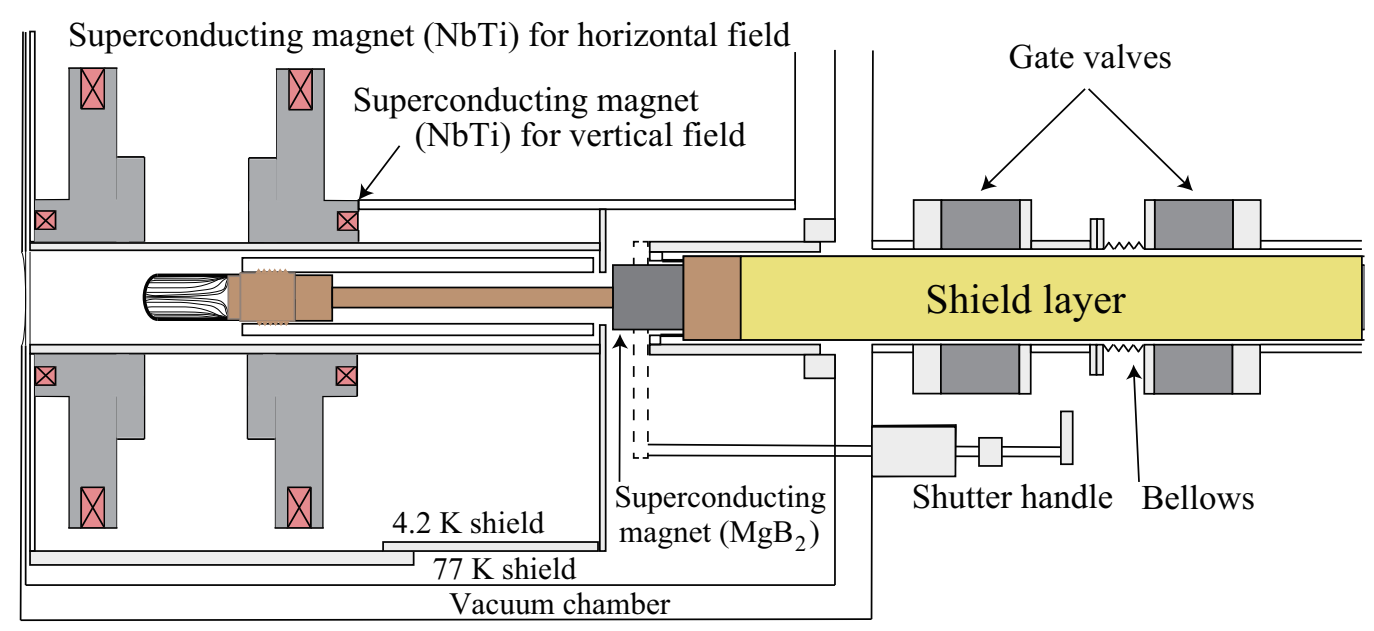

Figure 5: The cross sectional view of the IBC and the TC2 when they are connected.

preamp, a tune circuit and a coil. The laptop PC includes the LabVIEW software. The PXI system contains a signal generator and an ADC. The coil dose not contain hydrogen for coating.

\subsection{Polarization and Relaxation Time}

We measured the polarization and relaxation time of the polarized solid HD target in 2015. The polarization of hydrogen was $44 \pm 1 \%$ and the relaxation time was $239 \pm 66$ days at $\mathrm{T}=\sim 0.3$ $\mathrm{K}, \mathrm{B}=0.9 \mathrm{~T}$ (the same condition of the experiment).

We also observed polarization growing of hydrogen by using the Portable NMR system at $17 \mathrm{~T}$ [I4]]. The result of relaxation time of hydrogen was 5 hours at $\sim 30 \mathrm{mK}$. If there are too much ortho- $\mathrm{H}_{2}$, the HD is easy to be polarized, but the aging period becomes longer. If the amount of ortho- $\mathrm{H}_{2}$ is small, the HD is hard to be polarized. We would like to determine optimal amount of ortho- $\mathrm{H}_{2}$ for efficiently polarizing the HD. This study can determine optimal amount of ortho- $\mathrm{H}_{2}$.

\subsection{Status of the transportation}

We have succeeded in connecting the DR and the TC1, the TC1 and the SC also were connected at $4.2 \mathrm{~K}$ in RCNP before 2017. The SC and the TC2 were connected at room temperature and atmospheric pressure in summer of 2017 . We will try it at $77 \mathrm{~K}$ and vacuum pressure next. The TC2 and the IBC were connected at $77 \mathrm{~K}$ and vacuum pressure in September of 2016, after that we connected the TC2 and the IBC at $4.2 \mathrm{~K}$ and vacuum pressure in summer of 2017. We will excite the superconducting magnet of each cryostat when the all cryostats are connected at low temperature and vacuum pressure.

\section{Summary}

The polarization of the solid HD target obtained until now was $44 \%$ and a relaxation time was about 8 months in the same condition as that of the future experiment at SPring- 8 . The growth of 
the Hydrogen polarization have successfully been monitored, so that we will be able to determine the optimal amount of the ortho- $\mathrm{H}_{2}$. The IBC and the TC2 connection has been succeeded at the LHe temperature. The TC2 and the SC connection has been succeeded at room temperature. The connection of the TC2 and the SC will be done at low temperature soon. After succeeding all the target transportations, we will start the physics experiment at SPring-8.

\section{Acknowledgments}

We really appreciate the staff of the SPring- 8 facility for the supports. The experiments will be carried out at the BL33LEP of SPring-8 with the approval of the Japan synchrotron Radiation Research Institute (JASRI) as a contract beam line (Proposal No. BL33LEP/6004). This research was supported in part by the Ministry of Education, Science, Sports and Culture of Japan, the National Science Council of the Republic of China.

\section{References}

[1] H. Kohri, S. Y. Wang, S. H. Shiu, et al., Differential cross section and photon-beam asymmetry for the $\vec{\gamma} p \rightarrow \pi^{+} n$ reactions at forward $\pi^{+}$angles at $E_{\gamma}=1.5-2.95 \mathrm{GeV}$, Phys. Rev. C 97, (2018) 015205

[2] K. Mizutani, M. Niiyama, T. Nakano, et al., $\phi$ photoproduction on the proton at $E_{\gamma}=1.5-2.9 \mathrm{GeV}$, Phys. Rev. C 96, (2017) 062201(R)

[3] CLAS collaboration, D. Ho et al., Beam-Target Helicity Asymmetry for $\vec{\gamma} \vec{n} \rightarrow \pi^{-} p$ in the $N^{*}$ Resonance Region, Phys. Rev. Lett. 118, (2017) 242002

[4] LEGS-Spin Collaboration, S. Hoblit et al., Measurements of $\vec{H} \vec{D}(\vec{\gamma}, \pi)$ and Implications for the Convergence of the Gerasimov-Drell-Hern Integral, Phys. Rev. Lett. 102, (2009) 172002

[5] A. I. Titov, Y. Oh, S. N. Yang, Polarization Observables in $\phi$-Meson Photoproduction and the Strangeness Content of the Proton, Phys. Rev. Letters 79 (1997) 1634

[6] M. Bassan, S. Bouchigny, C. Commeaux, J.-P. Didelez et al., Static and dynamic polarization of HD, Nucl. Instr. Methods Phys. Res. A 526 (2004) 163-167.

[7] A. Honig, Highly Spin-Polarized Proton Samples -Large, Accessible, and Simply produced, Phys. Rev. Lett. 19, (1967) 1009

[8] M. Rigney, M. Breuer, S. Buhler, C. Commeaux et al., Solid HD polarized target: conceptual design of transport and in-beam cryostat, Nucl. Instr. Methods Phys. Res. A 356 (1995) 116-119.

[9] Xiangdong Wei, Christopher M. Bade, Anthony Caracappa, Tsuneo Kageya et al., New improvements leading to higher polarization frozen spin HD targets at the LEGS facility, Nucl. Instr. Methods Phys. Res. A 526 (2004) 157-162.

[10] S. Bouchigny, C. Commeaux, J.-P. Didelez, G. Rouillé, Progress in the polarization of HD, Nucl. Instr. Methods Phys. Res. A 544 (2005) 417-420.

[11] T. Ohta et al., Distillation of hydrogen isotopes for polarized HD target, Nucl. Instr. Methods Phys. Res. A 664 (2012) $347-352$.

[12] S. Bouchigny, J.-P. Didelez, F. Dubois, G. Rouille, Distillation of HD gas and measurement of spin-lattice relaxation times, Nucl. Instr. Methods Phys. Res. A607 (2009) 271-278. 
[13] T. Ohta, et al., Development of portable NMR polarimeter system for polarized HD target, Nucl. Instr. Methods Phys. Res. A633 (2011) 46-50.

[14] T. Ohta, et al., Development of 17T-NMR system for measurement of polarized HD and 3He targets, PoS (PSTP2015) 020 